\title{
Molecular insights into the mechanisms of M-cell differentiation and transcytosis in the mucosa-associated lymphoid tissues
}

\author{
Shunsuke Kimura ${ }^{1}$ (D)
}

Received: 28 September 2017 / Accepted: 13 October 2017 / Published online: 2 November 2017

(C) Japanese Association of Anatomists 2017

\begin{abstract}
Microfold cells (M cells), which are located in the follicle-associated epithelium (FAE) covering mucosal lymphoid follicles, are specialized epithelial cells that initiate mucosal immune responses. These cells take luminal antigens and transport them via transcytosis across the FAE to the antigen-presenting cells underneath. Several intestinal pathogens exploit $\mathrm{M}$ cells as their portal for entry to invade the host and cause disease conditions. Recent studies have revealed that the uptake of antigens by $\mathrm{M}$ cells is essential for efficient antigen-specific IgA production and that this process likely maintains the homeostasis of mucosal tissues. The present article reviews recent advances in understanding the molecular mechanism of M-cell differentiation and describes the molecules expressed by $\mathrm{M}$ cells that are associated with antigen uptake and/or the transcytosis process. Current efforts to augment M-cell-mediated uptake for use in the development of effective mucosal vaccines are also discussed.
\end{abstract}

Keywords RANK ligand - Peyer's patches · Microfold cells $\cdot$ Mucosal vaccine $\cdot$ Nasal-associated lymphoid tissue

\section{Introduction}

The mucosal surfaces of the gastrointestinal, respiratory, and urogenital tracts represent vast surface areas covered by epithelia, and they are exposed to a huge variety of ingested

Shunsuke Kimura

skimu@med.hokudai.ac.jp

1 Laboratory of Histology and Cytology, Graduate School of Medicine, Hokkaido University, North 15, West 7, Kita-ku, Sapporo 060-8638, Japan and inhaled antigens. Mucosal surfaces are protected by secretory products such as mucins, defensins, and antibodies-especially secretory immunoglobulin A (SIgA) —and by epithelial specializations that include tight junctions and apical surface glycocalyx. SIgA is the most abundant class of antibodies found in the mucosal surfaces of humans and most other mammals, and it plays an important role in the protection and homeostatic regulation of mucosal epithelia (Mantis et al. 2011).

The mucosa-associated lymphoid tissue (MALT) structures are the main sources of IgA-producing plasma cells in mucosal tissues. MALT is situated along the surfaces of all mucosal tissues. Its well-known representatives are gutassociated lymphoid tissue (GALT), nasopharynx-associated lymphoid tissue (NALT), and bronchus-associated lymphoid tissue (BALT) (Brandtzaeg et al. 2008). Although there are many differences between the MALTs in various organs, they all contain the same basic compartments-follicles, interfollicular regions, subepithelial dome regions, and follicle-associated epithelium (FAE). Notably, MALTs are nonencapsulated lymphoid organs that do not possess the afferent lymphatics that can serve as entry sites for lymphocytes and antigen-presenting cells with foreign antigens. Instead, the FAE can transport antigens from the mucosal lumen into the underlying lymphoid tissues. The FAE is characterized by the presence of microfold or membranous epithelial cells, termed $\mathrm{M}$ cells, which lack a typical brush border and possess a thin glycocalyx that provides better accessibility to large particulate antigens (Mabbott et al. 2013).

$M$ cells serve as entry sites of luminal antigen for the induction of efficient immune responses. $M$ cells are actively pinocytic cells that transport macromolecules and microorganisms from the intestinal lumen into the subepithelial region by a transepithelial transport system known as transcytosis. The antigen-presenting cells residing in the 
subepithelial region of the FAE subsequently collect the transcytosed macromolecules and microorganisms. In the absence of $\mathrm{M}$ cells or antigen sampling by $\mathrm{M}$ cells, antigenspecific T cell responses against orally infected Salmonella typhimurium and Yersinia enterocolitica are reduced in the Peyer's patches of mice, resulting in diminished levels of fecal antigen-specific SIgA production (Hase et al. 2009a, b; Kanaya et al. 2012; Kishikawa et al. 2017). Mice deficient in intestinal $\mathrm{M}$ cells have profound delays in the germinal center maturation of Peyer's patches and in the emergence of IgA plasma cells in the lamina propria of the gut. Consequently, the maturation of endogenous SIgA production during the weaning stage becomes delayed (Rios et al. 2016).

On the other hand, $\mathrm{M}$ cells are exploited by several different pathogenic bacteria, toxins, and prion proteins as a route of entry across the epithelial barrier into deeper tissues of the host. In the genetic absence of M cells in mice, the accumulation of prions within Peyer's patches and the spread of disease to the brain are blocked (Donaldson et al. 2016). Conversely, the prion uptake from the gut lumen is enhanced in mice that have been treated with RANKL, which increases $M$ cell density, resulting in shortened survival times. Therefore, the transcytosis of luminal antigens via $M$ cells is presumably an initial step in the induction of efficient immune responses to certain antigens and is important for the establishment of mucosal homeostasis. Furthermore, the regulatory mechanisms controlling M cell transcytosis are essential for maintaining the epithelial barrier at the mucosal surface.

The functions of $\mathrm{M}$ cells in mucosal immunity have attracted the attention of researchers because of their expected importance for regulating the mucosal immune systems and the wide potential applications that arise if it is possible to influence this system. For example, the targeted delivery of antigens to $\mathrm{M}$ cells has been recognized as an approach to enhancing oral vaccine efficacy (Devriendt et al. 2012). Still, it is only recently that the molecular basis for $\mathrm{M}$ cell differentiation and the transcytosis process have been uncovered. In this review, we focus on the molecular mechanisms of M-cell differentiation and transcytosis, as research has made rapid strides in these fields in recent years.

\section{The molecular mechanisms of M-cell differentiation}

\section{RANKL-RANK signaling is an essential factor in M-cell differentiation}

The TNF superfamily member receptor activator of NF- $\kappa B$ (RANK) and its ligand (RANKL) were originally discovered as a dendritic cell survival factor (Wong et al. 1997) and as an essential factor for bone homeostasis through the regulation of osteoclasts (Lacey et al. 1998; Yasuda et al. 1998). Williams and colleagues found that the RANKL-RANK pathway also regulates the differentiation of M cells in the FAE of GALT (Knoop et al. 2009). RANKL-null mice have hardly any M cells in their Peyer's patches and have a markedly diminished uptake of luminal latex beads compared with wild-type mice. This M-cell deficit can be recovered by the systemic administration of exogenous RANKL. Furthermore, the treatment of these mice with recombinant RANKL also induced the ectopic differentiation of villous $M$ cells on intestinal villi with the capacity for microorganism and fluorescent bead uptake.

All intestinal epithelial cell lineages develop from epithelial stem cells residing in the crypts. Each of the domeassociated crypts surrounding the FAE and the villous crypts at the base of the villi contain cycling leucine-rich repeatcontaining $\mathrm{G}$ protein-coupled receptor (Lgr5)-positive stem cells (Barker et al. 2007). Clevers and his colleagues performed a genetic lineage tracing study using Lgr5-promoter activity and showed that $\mathrm{M}$ cells derive from these cryptbased Lgr5 stem cells, as do other intestinal epithelial cell populations, including enterocytes, goblet cells, enteroendocrine cells, tuft cells, and Paneth cells (de Lau et al. 2012). These researchers also established an in vitro culture system of isolated single crypts that was devoid of mesenchymal cells and immune cells and contained only the minimal elements required for the maintenance and proliferation of the stem cells (Sato et al. 2009). The cultured crypts faithfully mimicked in vivo multiple crypt fission and differentiation events, and they eventually gave rise to organoids with protruding crypts and spherical villus-like epithelial domains containing all the differentiated cell types. When RANKL was added to this organoid culture system grown from isolated mouse crypts, some cells in the villus-like epithelial domains became M cells (de Lau et al. 2012). This phenomenon is identical to that observed in the corresponding human organoid culture system (Rouch et al. 2016). Taken together, these findings reveal that RANKL is a critical inducer for the differentiation of Lgr5-expressing intestinal stem cells into $\mathrm{M}$ cells.

\section{Subepithelial mesenchymal cells are the main inducers of M-cell differentiation}

In GALTs, RANKL is expressed in a variety of cell types, including group 3 innate lymphoid cells (ILC3 s), T cells, and mesenchymal cells (Cella et al. 2010; Totsuka et al. 2009; Taylor et al. 2007). Nagashima et al. (2017) first identified the M-cell inducer cells (MCi cells), which are podoplanin ${ }^{+}$subepithelial mesenchymal cells. MCi cells abundantly express RANKL on their cell surfaces and directly interact with the FAE. These researchers further showed that the genetic deletion of RANKL in a 
mesenchymal cell lineage impaired both M-cell differentiation and M-cell-dependent antigen sampling. Meanwhile, the expression levels of $\mathrm{M}$ cell-associated genes were normal in the FAE of GALTs from Tnfsf $11^{\mathrm{fl} / \Delta}$; Vav-iCre mice, in which RANKL is specifically deleted in the hematopoietic cells (including ILC $3 \mathrm{~s}$ and T cells), indicating that the RANKL expressed on T cells and ILC3s is dispensable for M-cell differentiation. These data suggest that the subepithelilal mesenchymal cells serve as M-cell inducers.

\section{Downstream process of the RANKL-RANK pathway in M-cell differentiation}

\section{The alternative pathway of $N F-\kappa B$ signaling regulates the initiation of $M$-cell differentiation}

The interaction of RANKL with its receptor, RANK, the cytoplasmic domain of which binds TRAF family proteins, transmits signals to downstream targets, mainly the NF- $\mathrm{kB}$ pathway (Anderson et al. 1997). NF-KB signaling can occur through two distinct pathways, the classical (canonical) and alternative (noncanonical) pathways, which share a common pattern: dimers of the transcription complex are retained in the cytoplasm in a latent inactive form until they are activated and rapidly transported into the nucleus (Oeckinghaus et al. 2011). In the classical NF- $\kappa B$ pathway, IKK $\beta$ phosphorylates $\mathrm{I} \kappa \mathrm{B} \alpha$, leading to its ubiquitination and proteolysis. The degradation of I $\kappa \mathrm{B} \alpha$ releases NF- $\kappa \mathrm{B}$ complexes (predominantly p65:p50 dimers) for nuclear translocation. This classical NF- $\kappa B$ pathway is activated within minutes, is usually transient, and is independent of de novo protein translation.

The alternative NF- $\kappa \mathrm{B}$ pathway is activated by IKK $\alpha$ following its phosphorylation by NF- $\mathrm{kB}$-inducing kinase (NIK). The phosphorylation of IKK $\alpha$ results in the processing of p100 to p52 via limited degradation in the proteasome and the subsequent formation of RelB:p52 heterodimers for nuclear translocation. Activation of this alternative NF- $\mathrm{kB}$ pathway requires more time (several hours) than is needed for activation of the classical NF- $\mathrm{KB}$ pathway (several minutes), is typically sustained for several days, and requires nascent protein synthesis. The classical NF- $\mathrm{kB}$ pathway is induced, at least to some extent, by all NF- $\mathrm{\kappa B}$-activating stimuli, whereas triggers of the alternative NF- $\kappa B$ pathway are limited to a subset of the TNF family of cytokines; this activation occurs efficiently in response to RANKL, B-cell activating factor (BAFF), CD40L, TWEAK, or lymphotoxin- $\beta$ (LT $\beta$ ) but not tumor necrosis factor $\alpha$ $(\mathrm{TNF}-\alpha)$.

Exogenous RANKL administration to mice is able to induce ectopic M-cell differentiation in the intestinal epithelium (Knoop et al. 2009). In this situation, p100 is processed to p52 by limited degradation, and RelB is transported into the nucleus within one day after RANKL administration; however, the amounts of p50 and nuclear p65 are not changed (Kimura et al. 2015a). Alymphoplasia (aly/aly) mice have a point mutation in the Map3k14 gene causing a loss of function of NIK; accordingly, in these mice, RelB:p52 heterodimers are retained in the cytosol in their inactive form (Shinkura et al. 1999). RANKL administration to the aly/aly mice fails to induce the ectopic induction of $\mathrm{M}$ cells in the villus epithelium (Kimura et al. 2015a). Thus, M-cell differentiation is presumably regulated by the alternative NF- $\mathrm{KB}$ signaling that is mediated by RelB:p52 heterodimer nuclear translocation.

TNF- $\alpha$ enhances RANKL-induced M-cell-associated gene expression: implications of crosstalk between the canonical and alternative $N F-\kappa B$ pathways

The in vitro organoid culture system of intestinal crypts is very useful for studying signaling pathways involved in M-cell differentiation and for researching the influence of other cytokines on RANKL-induced differentiation. Addition of the cytokine TNF- $\alpha$ with RANKL in the organoid culture enhances the expression levels of multiple M-cellassociated genes, while TNF- $\alpha$ alone has little, if any, effect on the expression of M-cell-associated genes (Wood et al. 2016). It is well established that TNF- $\alpha$ activates the classi-

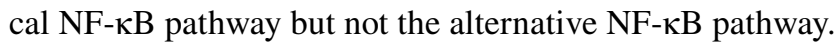
This effect is probably due to the ability of classical NF- $\mathrm{KB}$ activation to rapidly induce the enhanced expression of the genes encoding RelB and p100/p52, Relb and $N f \kappa b 2$, respectively. Consequently, the presence of more RelB and p100 proteins increases the number of potential RelB:p52 heterodimers, which may enhance the downstream effects of the RANKL-dependent alternative NF- $\mathrm{KB}$ signaling. Previous work supports this hypothesis; the transcriptional regulation of Relb and $N f \kappa b 2$ genes is dependent on the classical NF- $\kappa \mathrm{B}$ signaling pathway and constitutive p65 activity (Basak et al. 2008). LT $\beta R$ signaling activates first p65:p50 and then RelB:p52; furthermore, the first phase of p65 activity is required to induce p100 synthesis and subsequent RelB:p52 activity. Indeed, activation of RelB:p52 dimers by LT $\beta$ signaling is diminished in embryonic fibroblasts from p65-deficient mice. These studies indicate that interconnections between the classical and alternative NF- $\mathrm{KB}$ pathways exist, and these interconnections may be associated with the augmentation of M-cell differentiation.

Spi-B is an essential transcription factor for the early stage of $M$-cell differentiation

The regulation of M-cell differentiation by the ETS (E26 transformation-specific) transcription factor Spi-B was discovered independently by three research groups around the 
same time (Kanaya et al. 2012; de Lau et al. 2012; Sato et al. 2013). The expression of Spi-B in M cells was detected continuously from the crypts to the top of the FAE dome. Pulse chase experiments with 5-ethynyl-2'-deoxyuridine (EdU) labeling, a thymidine analogue that is incorporated into the DNA of dividing cells, revealed that, among the known M-cell-associated molecules, Spi-B is expressed from the earliest stage (Kimura et al. 2015a). The in vivo administration of RANKL to mice and its administration in the organoid culture system grown from the intestinal crypts both rapidly induce Spib gene expression in the epithelium (Kanaya et al. 2012; Lau et al. 2012). Furthermore, in the Spi-B-deficient mice, M cells are entirely absent in the FAE of Peyer's patches (Kanaya et al. 2012; de Lau et al. 2012; Sato et al. 2013). Therefore, Spi-B appears to regulate the early stage of M-cell differentiation.

Transcription of the mouse Spib gene can be initiated by two distinct promoters, yielding transcript variants designated Spib-1 and Spib-2 (Chen et al. 1998). The Spib-1 promoter contains a consensus NF-кB-binding site upstream of a TATA box sequence located just $5^{\prime}$ to the transcriptional start site. The Spib-2 promoter includes a recognition site for octamer transcription factors but does not include a TATA box. In the organoid culture system, RANKL exclusively induces the Spib-1 transcript; Spib-2 mRNA is not induced by the addition of RANKL (Wood et al. 2016). This RANKL-induced Spib expression requires NIK activation. Thus, these data suggest that Spib is a target gene of RANKL-NIK-NF- $\mathrm{BB}$ signaling.

\section{Two subsets of intestinal $M$ cells with distinct antigen uptake capacities}

Following the RANKL-stimulation-induced commitment of crypt stem cells to the M-cell lineage, these cells migrate along the crypt-FAE axis toward the top of the FAE. M cells in the FAE are further classified into two functionally different subtypes based on the expression of glycoprotein 2 (GP2) (Kimura et al. 2015a). GP2-high M cells actively take up luminal microbeads, whereas GP2-negative or -low cells scarcely ingest them. Both subsets of $\mathrm{M}$ cells share a Ulex europaeus agglutinin I (UEA-I) lectin-binding property as well as morphological features that are characteristic of $\mathrm{M}$ cells, and both subsets equally express other M-cell signature genes, including Spib, Tnfaip2, Ccl9, Pgryrp1, and Anxa5. Whole-mount immunohistochemistry combined with EdU pulse labeling revealed that the expression period of GP2 in the M cells appears later than that of Spi-B and Tnfaip2. These data suggest that GP2-high cells represent matured and functional $\mathrm{M}$ cells.

By analyzing the proportion of GP2-high M cells, we previously showed that the M-cell maturation level differs among GALTs located in different regions as well as among different mouse strains (Kimura et al. 2015a). We found that GP2-high M cells are scarce in the cecal patches of C57BL/6 mice, while GP2-low M cells are predominant there. Consistently, the cecal M cells in C57BL/6 mice showed only minimal uptake of luminal microbeads. Furthermore, in $\mathrm{BALB} / \mathrm{c}$ mice, the proportion of GP2-high $\mathrm{M}$ cells in the cecal patches was $\sim 50 \%$; this amount was much higher than that in C57BL/6 mice ( 1\%), but it was the lowest GP2high M-cell proportion among the GALTs of BALB/c mice. The induction of ectopic GP2-high M cells by GST-RANKL treatment was also suppressed in the cecal FAE of BALB/c mice. These data reveal that the M-cell maturation process is commonly suppressed in the cecum in both mouse strains.

The suppression of M-cell differentiation described above was most likely due to a decrease in the nuclear translocation and/or retention of RelB (Kimura et al. 2015a). A quantitative image analysis in C57BL/6 mice also demonstrated that, in contrast to the ileal M cells, which abundantly express nuclear RelB, a major population of cecal M cells in C57BL/6 mice did not sufficiently express nuclear RelB, resulting in GP2-negative or -low immature M cells. However, the proportion of RelB-positive $\mathrm{M}$ cells was higher in the cecal patches of BALB/c mice than in those of C57BL/6 mice. That is, the RelB activity in M cells is correlated with the degree of their maturation. RelB is probably required for the initiation of M-cell differentiation, and it is also a key determinant of their maturation through its regulation of the induction of downstream genes.

Cecal patches, like Peyer's patches, have an adequate ability to induce antigen-specific immunoglobulin responses and generate IgA-secreting cells that migrate to the large intestine (Masahata et al. 2014). A huge number of commensal bacteria inhabit the cecum because the cecum in the mouse is a major site for microbial fermentation. Given that M-cell-dependent antigen uptake initiates an antigenspecific mucosal immune response, it is possible that the attenuated M-cell maturation in the cecal epithelium may prevent excessive antigen uptake and the subsequent immune response and, in this manner, contribute to establishing a mutualistic symbiosis with the commensal microbiota that abundantly colonize mouse ceca.

\section{The fundamental differentiation mechanisms of $M$ cells are conserved in nasopharynx-associated lymphoid tissue}

The nasal cavity in rodents develops mucosal lymphoid tissues, collectively called nasopharynx (or nasal)-associated lymphoid tissues (NALTs), that are nestled along both sides of the nasopharyngeal duct and are generally considered to correspond to Waldeyer's ring in humans. NALT serves as an inductive site for mucosal immunity in the upper respiratory tract. The FAE of NALT consists of pseudostratified 
ciliated columnar epithelium and includes $\mathrm{M}$ cells. The ability of NALT M cells to take up luminal antigens is considered to be similar to that of intestinal M cells (Gebert and Pabst 1999). Morphologically, the NALT M cells in mice are closely associated with intraepithelial lymphocytes; these $\mathrm{M}$ cells extend their characteristic stubby microvilli, which are approximately $300 \mathrm{~nm}$ thick, and short, irregular microfolds on the luminal surface (Fig. 1). In contrast, the surrounding cells are covered with long cilia and fine microvilli, with the latter being approximately $100 \mathrm{~nm}$ thick (Mutoh et al. 2016). No cilia have been detected on the surfaces of $M$ cells. Histochemically, NALT M cells have been identified by the expression of vimentin and cytokeratin 8,18 , and 20 in the rabbit palatine tonsil (Carapelli et al. 2004; Gebert et al. 1995; Jepson et al. 1992), by the expression of clusterin, class II $\beta$-tubulin, and cytokeratin 20 in the human Waldeyer's ring (Lee et al. 2010; Verbrugghe et al. 2008; Takano et al. 2008), and by affinity to UEA-I lectin in murine and rat NALT (Jeong et al. 1999; Kim et al. 2011).

In murine NALT, GP2, and Tnfaip2 double-positive cells $\left(\mathrm{GP} 2^{+}\right.$Tnfaip $^{+} \mathrm{M}$ cells) on the FAE exhibit morphological features typical of $\mathrm{M}$ cells, share the ability to take up luminal microbeads, and express additional molecules specific to intestinal M cells, including Spi-B and Ccl9 (Mutoh et al. 2016). The GP2 ${ }^{+}$Tnfaip2 $^{+}$cells in the nasal cavity therefore correspond to intestinal $\mathrm{M}$ cells. Furthermore, RANKL-RANK signaling initiates the differentiation of $\mathrm{M}$ cells in the ciliated epithelium covering NALT, as it does in the intestinal columnar epithelium. The downstream factors RelB and Spi-B are involved in regulating the subsequent differentiation process. Accordingly, the fundamental molecules expressed in $\mathrm{M}$ cells and the M-cell differentiation process are both likely common to NALT and GALT. This implicates the possibility that RANKL-RANK signaling may induce the differentiation of M cells in other MALTs in the conjunctiva, tear ducts, bronchi, and salivary gland ducts.

Unlike the intestine where GP2 expression is mainly restricted to the $\mathrm{M}$ cells of the FAE, numerous GP2expressing nonciliated cells have been found to be distributed in the epithelium covering the paranasal sinuses, tear ducts, and conjunctiva in the head region of mice (Fig. 2) (Kimura et al. 2014, 2015b). However, these cells do not express other M-cell markers, such as UEA-I lectin, Tnfaip2, Spi-B, and CCL9. Histochemical studies suggest that GP2-expressing cells in the paranasal sinuses and conjunctiva are a lineage of goblet cells because they are positive for periodic acid-Schiff staining (Kimura et al. 2014). Notably, GP2 alone may not serve as a specific marker for $M$ cells in the nasal and ocular tissues of mice.

Kim et al. have recently reported that UEA-I ${ }^{+} \mathrm{M}$ cells reside in the nasal respiratory epithelium (Kim et al. 2011). Although these UEA-I ${ }^{+}$M cells are not associated with any lymphoid tissues, they are able to take up respiratory pathogens and initiate an antigen-specific immune response under experimental conditions. GP $2^{+}$Tnfaip $2^{+}$ $\mathrm{M}$ cells are also reactive with UEA-I lectin; however, their localization is restricted to the NALT FAE (Mutoh et al. 2016). Our previous studies failed to detect any GP2 ${ }^{+}$ Tnfaip $2^{+}$cells or specific immunoreactivities for CCL9 and Spi-B in the respiratory area of the nasal mucosa, even after the administration of RANKL. A RANKL injection exclusively induces $\mathrm{GP}^{+}{ }^{+} \mathrm{Tnfaip}^{+} \mathrm{M}$ cells in the FAE but not in the ordinary epithelium of the respiratory area. This result is consistent with the limited localization of its receptor RANK to the FAE. Therefore, UEA-I' "M cells" outside of the NALT might differ from authentic M cells expressing GP2 and Tnfaip2, and their differentiation might be independent of RANKL-RANK signals.

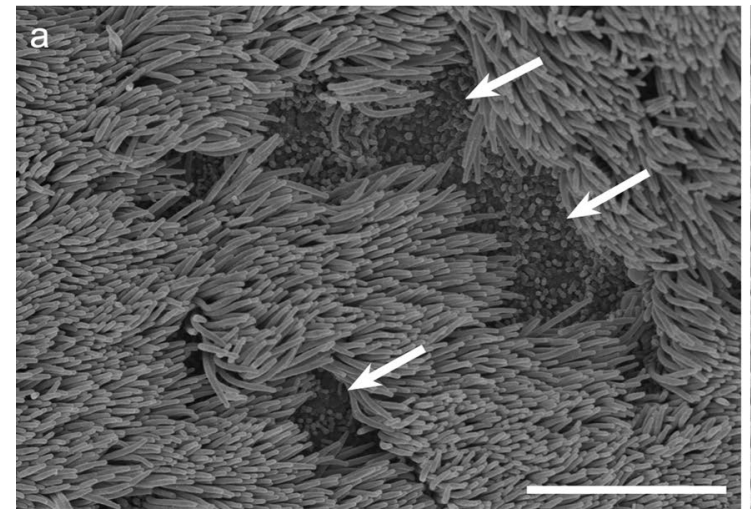

Fig. 1 Scanning electron micrograph of the luminal surface of NALT. a M cells (arrows) are intermingled among ciliated cells. b High magnification of an $M$ cell displaying thick stubby microvilli

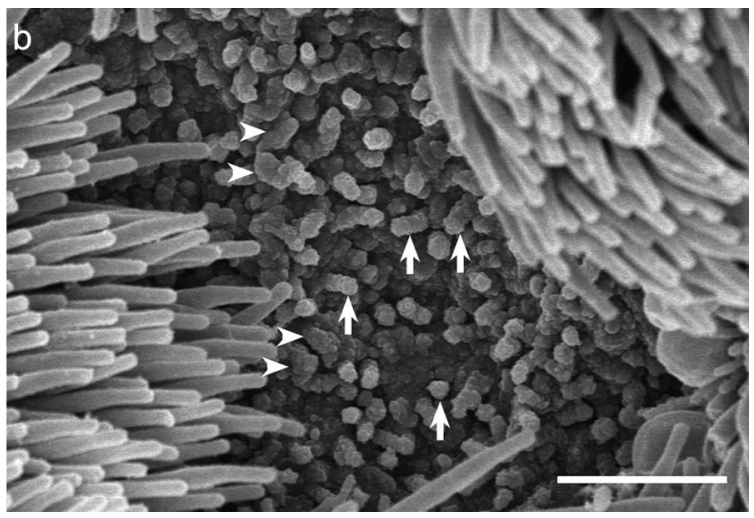

(arrows) and short microfolds (arrowheads). Bars: $10 \mu \mathrm{m}$ (a) and $5 \mu \mathrm{m}(\mathbf{b})$. This figure is reprinted with permission from Mutoh et al. (2016) 
A

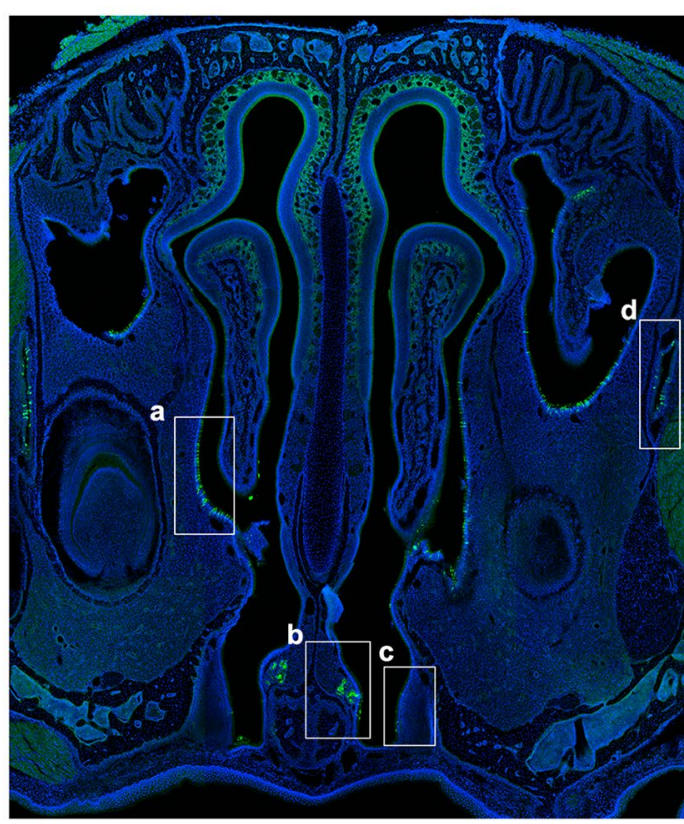

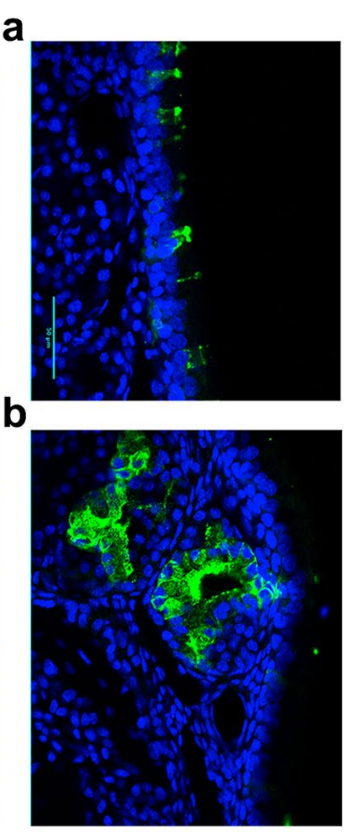

C

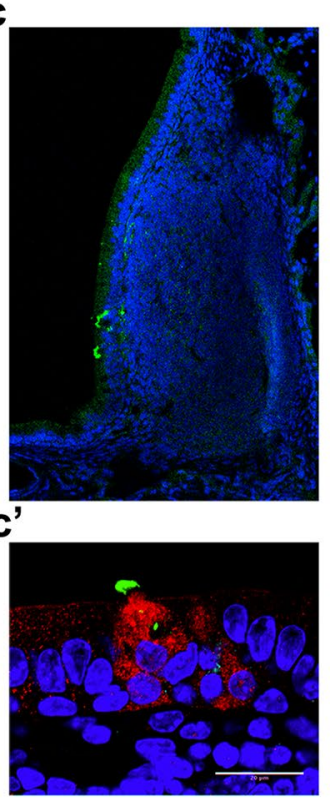

d

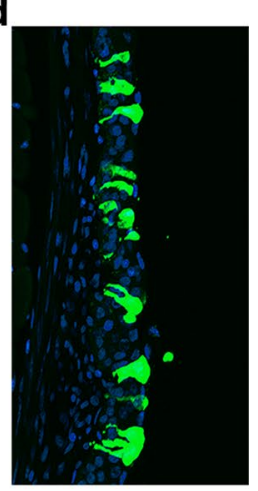

B

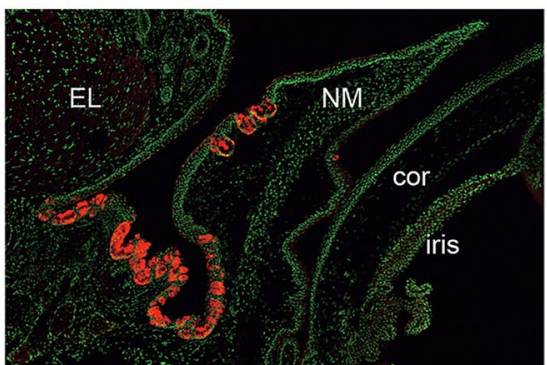

Fig. 2 Distribution of GP2-positive cells in the heads of mice (a). Immunohistochemical staining with anti-GP2 antibody on a coronal section of the head of a mouse. GP2-positive cells in the epithelium covering the paranasal sinuses $(a)$, the colleterial gland $(b)$, the NALT FAE $\left(c\right.$ and $\left.c^{\prime}\right)$, and the nasolacrimal gland $(e)$ are shown in green. GP2-positive cells on the NALT FAE expressing Tnfaip2, another M-cell signature gene, are shown in red $\left(c^{\prime}\right)$. Nuclei are stained with a nucleic acid dye, DAPI, which is shown in blue. Panel $c^{\prime}$ is reprinted

\section{Transcytosis}

$M$ cells have unique morphological features suited to efficient endocytosis and/or transcytosis. They lack the rigid brush border, and their apical surfaces possess poorly developed glycocalyx and undeveloped microvilli, which allow the cells to have easy access to the intestinal lumen to take up antigens. Furthermore, the M-cell basolateral membrane is deeply invaginated to form a large intraepithelial "pocket" that holds lymphocytes and mononuclear phagocytes. Due to this structural specialization, the basolateral cell surface is juxtaposed to the apical surface at intervals of a few microns; the distance narrows accordingly, making it easy for transcytotic vesicles to translocate through the epithelial barrier. Nonetheless, the molecular mechanisms of the with permission from Mutoh et al. (2016). b Immunohistochemical staining of GP2 in the conjunctiva of mice. GP2-positive cells gather or are scattered in the squamous stratified epithelium (left and middle panels). After imaging GP2 immunostaining (middle panel), the same section was stained for PAS reaction $(d)$. Nuclei are stained with a nucleic acid dye, SYTOX, which is shown in blue. cor cornea, $E L$ eyelid, $N M$ nictitating membrane. Panel $\mathbf{b}$ is reprinted with permission from Kimura et al. (2015b)

transcytosis process via $\mathrm{M}$ cells are still largely unknown. However, researchers have identified some molecules that are highly expressed on $\mathrm{M}$ cells that act as receptors on the cell surface for luminal microorganisms and are probably associated with vesicular transport as they regulate the cellular cytoskeleton and membrane compartments of $\mathrm{M}$ cells.

\section{Cell surface proteins on $\mathrm{M}$ cells may serve as receptors for foreign antigens}

GP2 is a well-characterized cell surface protein known to serve as a transcytotic receptor on $\mathrm{M}$ cells (Hase et al. 2009a). GP2 was originally discovered as an integral membrane protein that is secreted from the zymogen granules of pancreatic acinar cells and is associated with the plasma 
membrane via a glycosylphosphatidylinositol (GPI) linkage (Scheffer et al. 1980; Rindler and Hoops 1990). In the absence of GP2, the uptake and transcytosis of particular types of bacteria and toxins by $\mathrm{M}$ cells are severely impaired (Hase et al. 2009a; Matsumura et al. 2015), suggesting that GP2 serves as an uptake receptor for a subset of commensal and pathogenic bacteria on M cells. In fact, GP2 interacts with FimH, a major component of the type 1 pilus on the outer membrane of a subset of Gram-negative enterobacilli such as Escherichia coli and Salmonella typhimurium, and a GP2-FimH interaction is necessary for the efficient uptake of FimH ${ }^{+}$bacteria by $\mathrm{M}$ cells and the subsequent bacteriaspecific mucosal immune responses (Hase et al. 2009a).

Botulinum neurotoxin (BoNT), which is produced by Clostridium botulinum and related species, is a potent metalloprotease toxin that acts on neuronal cells and causes foodborne botulism. On its own, BoNT is not absorbed by the intestinal epithelium and has little oral toxicity. However, BoNT is naturally produced in the form of a large progenitor toxin complex (L-PTC), which is a multiprotein complex composed of BoNT, nontoxic nonhemagglutinin, and hemagglutinin (HA). Remarkably, the oral toxicity of the L-PTC is hundreds of fold higher than that of the free toxin (Ohishi et al. 1977; Sakaguchi 1982; Cheng et al. 2008). The translocation route of botulinum toxin A through the epithelium to the neuronal cells was largely unknown; however, recent work proposed that $\mathrm{M}$ cells are the major site for the intestinal translocation of L-PTC (Matsumura et al. 2015). Susceptibility to orally administered L-PTC is dramatically reduced in mice that lack intestinal $\mathrm{M}$ cells due to treatment with anti-RANKL antibody. The HA in L-PTC interacts with the GP2 on M cells, and this interaction mediates the traverse of BoNT through the intestinal epithelial barrier.

Similarly, cellular prion protein $\left(\operatorname{PrP}^{\mathrm{C}}\right)$ is expressed on the apical surface of $M$ cells and interacts with heat-shock protein 60 (Hsp60) of the Gram-negative bacterial species Brucella abortus, which causes brucellosis and is known to infect via the oral route (Nakato et al. 2012). The translocation of B. abortus into Peyer's patches after its oral administration is significantly reduced in $\mathrm{PrP}^{\mathrm{C}}$-deficient mice, suggesting that the $\operatorname{PrP}^{\mathrm{C}}$ on $\mathrm{M}$ cells serves as a major uptake receptor for $B$. abortus during oral infection.

Other molecules with binding capacity for bacterial components are expressed on the surfaces of $\mathrm{M}$ cells, but their functions in $\mathrm{M}$ cells are still unclear. Uromodulin, a molecule that is highly similar to GP2, binds E. coli with type 1 fimbriae (Sato et al. 2013; Kuriyama and Silverblatt. 1986). Siglec-F is a sialic acid-specific lectin that has been shown to be a cellular receptor for the sialylated microbe Neisseria meningitides (Gicheva et al. 2016; Jones et al. 2003). Peptidoglycan recognition protein 1 (Pglyrp1), also known as PGRP-S, interacts with peptidoglycan in the bacterial cell wall (Lo et al. 2003; Dziarski et al. 2003).
The existence of cell surface molecules that serve as specific receptors demonstrates that $\mathrm{M}$ cells have a receptormediated transcytosis system for luminal antigens in addition to their nonselective antigen uptake process. The receptors on $\mathrm{M}$ cells will be a pharmacological target for blocking bacterial or toxin invasion via $\mathrm{M}$ cells or for enhancing the immune response by targeting antigens that are specific to $\mathrm{M}$ cells.

\section{M cells express cytosolic proteins that likely regulate M-cell morphology and transcytosis}

Allograft inflammatory factor 1 (Aif1) was originally discovered as a gene expressed in chronically rejected cardiac allografts, and is also known as ionized calcium-binding adapter molecule 1 (Iba1). Aif1 is a 17-kDa hydrophilic polypeptide that contains a calcium-binding EF-hand domain and is mainly expressed in macrophages/microglias and neutrophils (Utans et al. 1995; Ito et al. 1998). Kishikawa et al. (2017) reported that M cells in the FAE of Peyer's patches express Aif1. They found that, in Aif1-deficient mice, the uptake ability of luminal particles and enteric commensal bacteria via $M$ cells is severely impaired, whereas Aif1 deficiency does not affect the development and fundamental ultrastructure of $\mathrm{M}$ cells. This group further showed that Aif1 is involved in the activation of $\beta 1$ integrin at the apical surface of M cells. $\beta 1$ integrin is a potential receptor for the $Y$. enterocolitica protein invasin; the $\beta 1$ integrin-invasin interaction mediates the effective entry of this pathogenic bacterium into the Peyer's patches (Isberg and Leong 1990; Grützkau et al. 1990; Clark et al. 1998). A deficit of $\beta 1$ integrin activation in Aif1-deficient mice appears to be responsible for the decreased subsequent induction of antigen-specific IgA immune responses in these mice (Kishikawa et al. 2017).

Aif1 exhibits F-actin binding and crosslinking activity in macrophages/microglia cells, and it regulates membrane ruffling and phagocytosis by interacting with the small GTPase Rac (Ohsawa et al. 2000). Because Rac2 mRNA is highly expressed in the M cells of FAE (Terahara et al. 2008), it seems likely that Aif1 may contribute to the regulation of $\mathrm{F}$-actin remodeling in $\mathrm{M}$ cells by cooperating with Rac2 for the uptake of particles and enteric commensal bacteria by $\mathrm{M}$ cells. During the phagocytosis of $Y$. enterocolitica, the interaction of invasin with $\beta 1$ integrin triggers the formation of phagocytic cups by activating actin polymerization through several small GTPases, including Rac, and their effectors (Wiedemann et al. $2001)$. Both Aif1 and Rac probably function downstream of $\beta 1$ integrin, but it is still unclear how Aif 1 regulates the activation of $\beta 1$ integrin. Nonetheless, the uptake of particles and some kinds of commensal bacteria via $\mathrm{M}$ cells seems to be diminished in Aif1-deficient mice; therefore, 
further analyses of the Aif1 in M cells will provide insight into the molecular mechanisms of M-cell transcytosis.

Tnfaip2, also known as M-Sec or B94, is a cytosolic protein expressed in $M$ cells, human umbilical vein endothelial cells (HUVEC), and myeloid lineage cells such as macrophages and dendritic cells (Hase et al. 2009b; Wolf et al. 1994; Chevrier et al. 2011). In macrophages, Tnfaip2 has a potent ability to cause membrane deformation and plays a central role in the formation of tunneling nanotubes (TNTs) in cooperation with the small GTPase RalA and the exocyst complex (Hase et al. 2009b). TNTs are a relatively recently recognized membrane structure defined as filopodia-like thin protrusions of the plasma membrane that physically connect remote cells (Kimura et al. 2012, 2013). The expression of Tnfaip2 is also increased in nasopharyngeal carcinoma tissue and inflamed endothelial cells (Chen et al. 2011; Barzilai et al. 2016). Furthermore, a siRNA-mediated depletion of Tnfaip2 reduces the amount of cell migration and invasion by nasopharyngeal carcinoma HK1 cells (Chen et al. 2011). In the inflamed endothelial cells, Tnfaip 2 protein is localized at the plasma membrane, and the depletion of this gene reduces polarized secretion of cytokines and chemokines, suggesting that Tnfaip2 mediates the exocytosis process (Barzilai et al. 2016).

Mutational and crystallographic analyses showed that the N-terminal polybasic region of Tnfaip2 directly binds phosphatidylinositol $(4,5)$-bisphosphate and phosphatidylinositol $(3,4,5)$-bisphosphate for its localization to the plasma membrane (Kimura et al. 2016). Tnfaip2 interacts with the RalA, and this interaction is essential for its membrane deformation ability. A crystal structure of M-Sec consists of helix bundles arranged in a straight rod-like shape, similar to that of membrane-tethering complex subunits such as Sec6, Exo70, and Tip20. A positively charged surface in the $\mathrm{C}$-terminal domains of Tnfaip2 is required for its interaction with active RalA to extend the plasma membrane protrusions, suggesting that the membrane-associated Tnfaip 2 recruits active RalA, which subsequently directs the exocyst complex to the site of membrane deformation.

Together, these data suggest that the Tnfaip2 protein cycles between cell membranes and the cytosol and that it may play a role in controlling cellular membrane morphology. In M cells, Tnfaip2 is mainly detected in the cytosol, but it is sometimes associated with the plasma membrane and co-localizes with incorporated microbeads (Hase et al. 2009b; Kimura et al. 2015a; Mutoh et al. 2016). Tnfaip2 is therefore presumably associated with M-cell morphology and the M-cell transcytosis/endocytosis process; however, additional experiments are required to determine its in vivo functions in $\mathrm{M}$ cells.

\section{Mucosal vaccine development}

Because the mucosal surface serves as an important site of initial contact and as a portal of entry for pathogens, it is critical to develop strategies for neutralizing pathogens at the mucosal surface. Mucosal vaccination via an oral or nasal route can lead to immune responses not only at the mucosal site of administration but also at other mucosal sites and/or systemic sites. Therefore, mucosal vaccines are expected to eventually become an effective preventive measure against various infectious diseases. Presently, however, the oral and nasal routes for vaccination are still challenging and difficult to achieve, and progress in mucosal vaccine development has been rather slow (Devriendt et al. 2012).

Our molecular understanding of M-cell biology has informed the design of oral and nasal vaccines with improved efficacy for priming mucosal and systemic immune responses. In fact, orally administered biotinylated ovalbumin peptide (OVA) conjugated with anti-GP2-streptavidin efficiently induces OVA-specific fecal IgA secretion in a mouse model (Shima et al. 2014). Additionally, GP2-binding peptide fused with green fluorescent protein (GFP) significantly increases the uptake of enhanced GFP (EGFP) by M cells and induces efficient mucosal and systemic immune responses measured at the level of antigen-specific serum and fecal antibodies, cytokine secretion, and lymphocyte proliferation (Khan et al. 2017). Oral administration of tetanus toxoid- or botulinum toxoid-conjugated NKM 16-2-4, which is an M-cell-specific monoclonal antibody, induces high-level, antigen-specific serum IgG and mucosal IgA responses (Nochi et al. 2007). In addition, an oral vaccine formulation of botulinum toxoid-conjugated NKM 16-2-4 induces protective immunity against a lethal challenge with botulinum toxin.

The combination of targeting M-cell antigen delivery and controlling M-cell density could potentially further improve the effect of a vaccine. Cho and colleagues identified an M-cell-homing peptide through the use of a bacteriophage display system and developed an M-cell-targeting antigen that is a fusion protein of the bacterial outer membrane protein $\mathrm{BmpB}$ with the $\mathrm{M}$-cell-homing peptide (M-BmpB) (Yoo et al. 2010; Maharjan et al. 2016). In mice systemically treated with RANKL to enhance M-cell density, oral immunization with $\mathrm{M}-\mathrm{BmpB}$ induces strong protective IgA and systemic IgG antibody responses against $\mathrm{BmpB}$ (Maharjan et al. 2016). The authors further confirmed that the systemic administration of RANKL did not severely affect bone resorption activity under their experimental conditions (Maharjan et al. 2016). However, RANKL-RANK signaling is known to be involved in a number of important biological processes, including bone homeostasis, inflammation, cell differentiation, and body temperature control (Walsh and Choi 2014; Okamoto et al. 2017; Hanada et al. 


\section{$\begin{array}{lll} & \text { GP2-low M cells } & \text { GP2-high M cell } \\ & \text { Lower uptake capacity } & \text { Higher uptake capacity }\end{array}$}

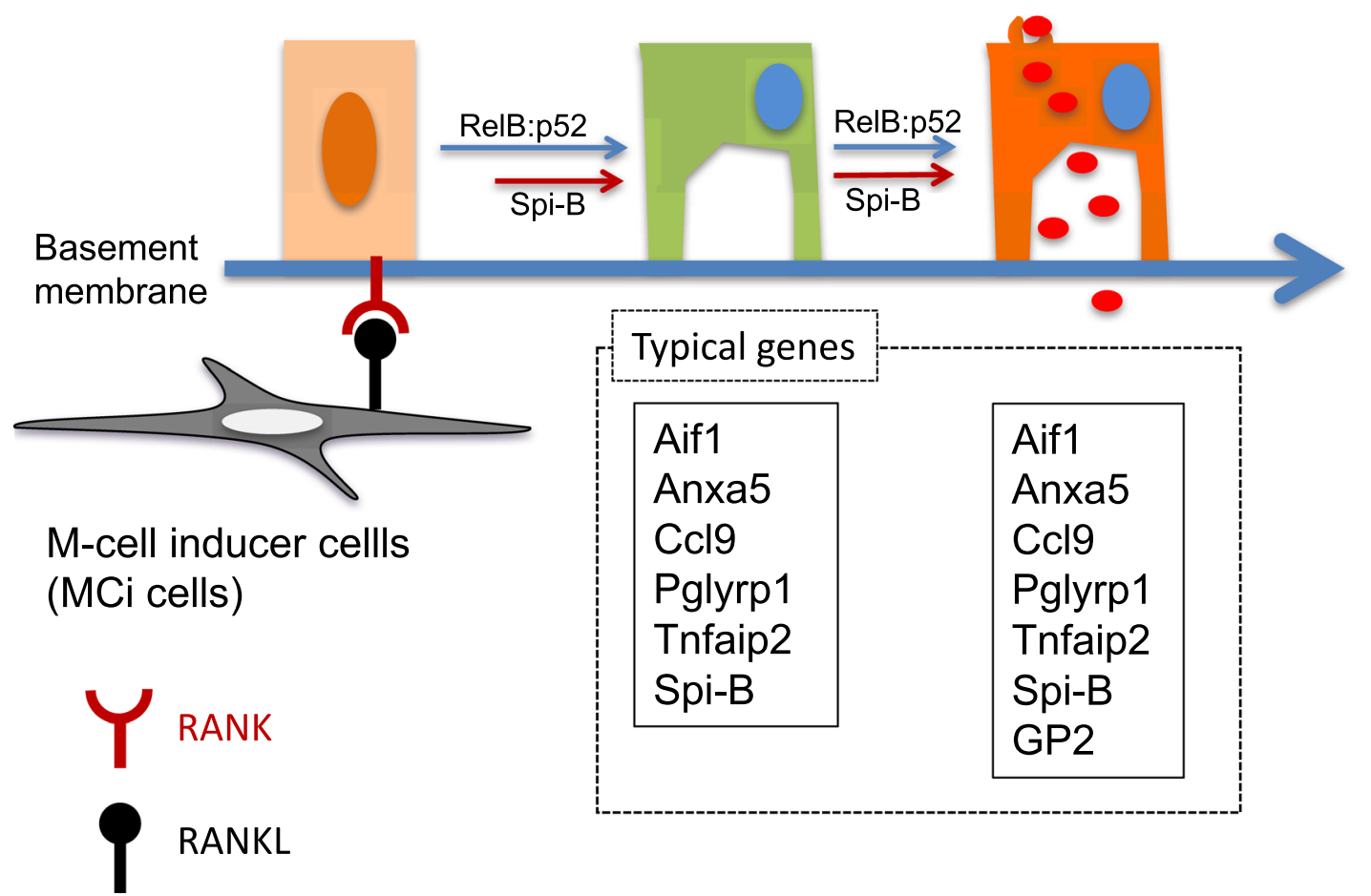

Fig. 3 Proposed model of M-cell differentiation. RANKL on the cell surface of podoplanin-expressing mesenchymal cells (M-cell inducer cells: MCi cells) interacts with its receptor (RANK) on the intestinal epithelial stem cells. This RANKL-RANK interaction triggers the nuclear translocation of RelB:p52 heterodimers to initiate M-cell differentiation. Spi-B is probably a target gene of RANKL-RelB:p52 signaling and is expressed at an early stage of the differentiation process. Spi-B regulates several M-cell signature genes. Differentiated

2009). Furthermore, M-cell density in the mucosal tissue is probably also associated with disease susceptibility. The invasions of intestinal pathogens, pathogenic products (toxins), and prion proteins into Peyer's patches are effectively blocked by neutralization antibodies against RANKL as well as within intestinal epithelial cell-specific RANKdeficient mice (Gonzalez-Hernandez et al. 2014; GonzalezHernandez et al. 2014; Donaldson et al. 2016; Matsumura et al. 2015). Consistently, the exogenous administration of RANKL to mice enhances prion uptake and toxin absorption from the oral route (Donaldson et al. 2016; Matsumura et al. 2015). Although systemic treatment with RANKL is a potent and attractive strategy because it can enhance the mucosal vaccine efficiency by increasing the number of $\mathrm{M}$ cells in the mucosal epithelium, its use will require the cautious consideration of potential side effects from enhancing the M-cell density and activating RANKL-RANK signaling in other organs.
$\mathrm{M}$ cells are classified into at least two functionally different subtypes based on the expression GP2. GP2-low M cells are immature M cells, and GP2-high M cells are mature $\mathrm{M}$ cells, which have a high uptake capacity for luminal antigens. The maturation from GP2-low M cells to GP2-high M cells also requires RelB:p52 activity. That is, ReB:p52 activity must be maintained during the entire process of M-cell differentiation and maturation

\section{Concluding remarks}

Accumulating knowledge from recent work about the molecular mechanisms underlying M-cell cellular differentiation has gradually outlined the main components of the M-cell differentiation process (Fig. 3). RANKL-triggered alternative NF- $\mathrm{KB}$ signaling is probably critical for the initiation of M-cell differentiation. In GALT, the subepithelial mesenchymal cells expressing RANKL (MCi cells) act on intestinal epithelial stem cells to direct their differentiation into $\mathrm{M}$ cells. The transcription factor Spi-B is required for their subsequent commitment to an M-cell fate. Further, differentiated $\mathrm{M}$ cells are classified into two functionally different subtypes based on the expression of GP2: GP2-high cells are mature $M$ cells and have a high uptake capacity, whereas GP2-negative or -low cells are immature M cells. The RANKL-mediated process is common to the GALT and NALT; therefore, this might be a fundamental mechanism 
underlying M-cell differentiation in the MALTs. Transcriptome studies of FAE have identified several M-cellexpressing genes that contain cell surface proteins serving as antigen receptors and cytosolic proteins which may be associated with the antigen uptake process and/or transcytosis. In conclusion, molecular-based knowledge of M-cell biology will contribute to the further understanding of mucosal immunity, which is likely to lead to improvements in mucosal vaccine efficiency.

Acknowledgements I thank Dr. Toshihiko Iwanaga, Professor of Hokkaido University, for his critical reading of the manuscript, and Katie Oakley, PhD, from Edanz Group (www.edanzediting.com/ac), for editing a draft of this manuscript. I wish to also thank the timely help given by Dr. Mami Mutoh, Dr. Megumi Yamakami-Kimura, and Dr. Hiromi Takahashi-Iwanaga, in our laboratory, as well as that afforded by Dr. Koji Hase, Professor of Keio University. This work was supported by JSPS KAKENHI (grant numbers 23790521, 25460261, and 16K08457). This work was also supported by grants from the Research Foundation for Opto-Science and Technology, the Mochida Memorial Foundation for Medical and Pharmaceutical Research, The Food Science Institute Foundation, SENSHIN Medical Research Foundation, The NOASTEC Foundation, and GSK Japan Research Grand 2015.

\section{Compliance with ethical standards}

Conflict of interest The authors disclose no conflicts of interest.

\section{References}

Anderson DM, Maraskovsky E, Billingsley WL, Dougall WC, Tometsko ME, Roux ER, Teepe MC, DuBose RF, Cosman D, Galibert L (1997) A homologue of the TNF receptor and its ligand enhance T-cell growth and dendritic-cell function. Nature 390:175-179

Barker N, van Es JH, Kuipers J, Kujala P, van den Born M, Cozijnsen M, Haegebarth A, Korving J, Begthel H, Peters PJ, Clevers H (2007) Identification of stem cells in small intestine and colon by marker gene Lgr5. Nature 449:1003-1007

Barzilai S, Blecher-Gonen R, Barnett-Itzhaki Z, Zauberman A, LebelHaziv Y, Amit I, Alon R (2016) M-sec regulates polarized secretion of inflammatory endothelial chemokines and facilitates CCL2-mediated lymphocyte transendothelial migration. J Leukoc Biol 99:1045-1055

Basak S, Shih VF, Hoffmann A (2008) Generation and activation of multiple dimeric transcription factors within the NF-kappaB signaling system. Mol Cell Biol 28:3139-3150

Brandtzaeg P, Kiyono H, Pand Pabst, Russell MW (2008) Terminology: nomenclature of mucosa-associated lymphoid tissue. Mucosal Imuunol 1:31-37

Carapelli A, Regoli M, Nicoletti C, Ermini L, Fonzi L, Bertelli E (2004) Rabbit tonsil-associated M-cells express cytokeratin 20 and take up particulate antigen. J Histochem Cytochem 52:1323-1332

Cella M, Otero K, Colonna M (2010) Expansion of human NK-22 cells with IL-7, IL-2, and IL-1beta reveals intrinsic functional plasticity. Proc Natl Acad Sci USA 107:10961-10966

Chen HM, Gonzalez DA, Radomska HS, Voso MT, Sun Z, Zhang P, Zhang DE, Tenen DG (1998) Two promoters direct expression of the murine Spi-B gene, an Ets family transcription factor. Gene 207:209-218
Chen LC, Chen CC, Liang Y, Tsang NM, Chang YS, Hsueh C (2011) A novel role for TNFAIP2: its correlation with invasion and metastasis in nasopharyngeal carcinoma. Mod Pathol 24:175-184

Cheng LW, Onisko B, Johnson EA, Reader JR, Griffey SM, Larson AE, Tepp WH, Stanker LH, Brandon DL, Carter JM (2008) Effects of purification on the bioavailability of botulinum neurotoxin type A. Toxicology 249:123-129

Chevrier N, Mertins P, Artyomov MN, Shalek AK, Iannacone M, Ciaccio MF, Gat-Viks I, Tonti E, DeGrace MM, Clauser KR, Garber M, Eisenhaure TM, Yosef N, Robinson J, Sutton A, Andersen MS, Root DE, von Andrian U, Jones RB, Park H, Carr SA, Regev A, Amit I, Hacohen N (2011) Systematic discovery of TLR signaling components delineates viral-sensing circuits. Cell 147:853-867

Clark MA, Hirst BH, Jepson MA (1998) M-cell surface beta1 integrin expression and invasin-mediated targeting of Yersinia pseudotuberculosis to mouse Peyer's patch M cells. Infect Immun 66:1237-1243

de Lau W, Kujala P, Schneeberger K, Middendorp S, Li VS, Barker N, Martens A, Hofhuis F, DeKoter RP, Peters PJ, Nieuwenhuis E, Clevers H (2012) Peyer's patch M cells derived from Lgr5(+) stem cells require SpiB and are induced by RankL in cultured "miniguts". Mol Cell Biol 32:3639-3647

Devriendt B, De Geest BG, Goddeeris BM, Cox E (2012) Crossing the barrier: targeting epithelial receptors for enhanced oral vaccine delivery. J Control Release 160:431-439

Donaldson DS, Sehgal A, Rios D, Williams IR, Mabbott NA (2016) Increased abundance of $\mathrm{M}$ cells in the gut epithelium dramatically enhances oral prion disease susceptibility. PLoS Pathog 2:e1006075

Dziarski R, Platt KA, Gelius E, Steiner H, Gupta D (2003) Defect in neutrophil killing and increased susceptibility to infection with nonpathogenic gram-positive bacteria in peptidoglycan recognition protein-S (PGRP-S)-deficient mice. Blood 102:689-697

Gebert A, Pabst R (1999) M cells at locations outside the gut. Semin Immunol Semin Immunol 11:165-170

Gebert A, Willfuhr B, Pabst R (1995) The rabbit M-cell marker vimentin is present in epithelial cells of the tonsil crypt. Acta Otolaryngol 115:697-700

Gicheva N, Macauley MS, Arlian BM, Paulson JC, Kawasaki N (2016) Siglec-F is a novel intestinal M cell marker. Biochem Biophys Res Commun 479:1-4

Gonzalez-Hernandez MB, Liu T, Payne HC, Stencel-Baerenwald JE, Ikizler M, Yagita H, Dermody TS, Williams IR, Wobus CE (2014) Efficient norovirus and reovirus replication in the mouse intestine requires microfold (M) cells. J Virol 88:6934-6943

Grützkau A, Hanski C, Hahn H, Riecken EO (1990) Involvement of $\mathrm{M}$ cells in the bacterial invasion of Peyer's patches: a common mechanism shared by Yersinia enterocolitica and other enteroinvasive bacteria. Gut 31:1011-1015

Hanada R, Leibbrandt A, Hanada T, Kitaoka S, Furuyashiki T, Fujihara H, Trichereau J, Paolino M, Qadri F, Plehm R, Klaere S, Komnenovic V, Mimata $\mathrm{H}$, Yoshimatsu $\mathrm{H}$, Takahashi N, von Haeseler A, Bader M, Kilic SS, Ueta Y, Pifl C, Narumiya S, Penninger JM (2009) Central control of fever and female body temperature by RANKL/RANK. Nature 462:505-509

Hase K, Kawano K, Nochi T, Pontes GS, Fukuda S, Ebisawa M, Kadokura K, Tobe T, Fujimura Y, Kawano S, Yabashi A, Waguri S, Nakato G, Kimura S, Murakami T, Iimura M, Hamura K, Fukuoka S, Lowe AW, Itoh K, Kiyono H, Ohno H (2009a) Uptake through glycoprotein 2 of $\mathrm{FimH}(+)$ bacteria by $\mathrm{M}$ cells initiates mucosal immune response. Nature 462:226-230

Hase K, Kimura S, Takatsu H, Ohmae M, Kawano S, Kitamura H, Ito M, Watarai H, Hazelett CC, Yeaman C, Ohno H (2009b) M-Sec promotes membrane nanotube formation by interacting with Ral and the exocyst complex. Nat Cell Biol 11:1427-1432 
Isberg RR, Leong JM (1990) Multiple beta 1 chain integrins are receptors for invasin, a protein that promotes bacterial penetration into mammalian cells. Cell 60:861-871

Ito D, Imai Y, Ohsawa K, Nakajima K, Fukuuchi Y, Kohsaka S (1998) Microglia-specific localisation of a novel calcium binding protein, Iba1. Brain Res Mol Brain Res 57:1-9

Jeong KI, Uetsuka K, Nakayama H, Doi K (1999) Glycoconjugate expression in follicle-associated epithelium (FAE) covering the nasal-associated lymphoid tissue (NALT) in specific pathogenfree and conventional rats. Exp Anim 48:23-29

Jepson MA, Mason CM, Bennett MK, Simmons NL, Hirst BH (1992) Co-expression of vimentin and cytokeratins in $\mathrm{M}$ cells of rabbit intestinal lymphoid follicle-associated epithelium. Histochem $\mathrm{J}$ 24:33-39

Jones C, Virji M, Crocker PR (2003) Recognition of sialylated meningococcal lipopolysaccharide by siglecs expressed on myeloid cells leads to enhanced bacterial uptake. Mol Microbiol 49:1213-1225

Kanaya T, Hase K, Takahashi D, Fukuda S, Hoshino K, Sasaki I, Hemmi H, Knoop KA, Kumar N, Sato M, Katsuno T, Yokosuka O, Toyooka K, Nakai K, Sakamoto A, Kitahara Y, Jinnohara T, McSorley SJ, Kaisho T, Williams IR, Ohno H (2012) The Ets transcription factor Spi-B is essential for the differentiation of intestinal microfold cells. Nat Immunol 13:729-736

Khan IU, Huang J, Liu R, Wang J, Xie J, Zhu N (2017) Phage displayderived ligand for mucosal transcytotic receptor GP-2 promotes antigen delivery to $\mathrm{M}$ cells and induces antigen-specific immune response. SLAS Discov 22:879-886

Kim DY, Sato A, Fukuyama S, Sagara H, Nagatake T, Kong IG, Goda K, Nochi T, Kunisawa J, Sato S, Yokota Y, Lee CH, Kiyono $\mathrm{H}$ (2011) The airway antigen sampling system: respiratory $\mathbf{M}$ cells as an alternative gateway for inhaled antigens. J immunol 186:4253-4262

Kimura S, Hase K, Ohno H (2012) Tunneling nanotubes: emerging view of their molecular components and formation mechanisms. Exp Cell Res 318:1699-1706

Kimura S, Hase K, Ohno H (2013) The molecular basis of induction and formation of tunneling nanotubes. Cell Tissue Res 352:67-76

Kimura S, Muto M, Hisamoto M, Zheng M, Iwanaga T (2014) A novel type of cells expressing GP2 in the respiratory epithelium of the paranasal sinuses in mice. Biomed Res 35:329-337

Kimura S, Yamakami-Kimura M, Obata Y, Hase K, Kitamura H, Ohno $H$, Iwanaga $T$ (2015a) Visualization of the entire differentiation process of murine $\mathrm{M}$ cells: suppression of their maturation in cecal patches. Mucosal Immunol 8:650-660

Kimura S, Yamashita M, Yamakami-Kimura M, Sato Y, Yamagata A, Kobashigawa Y, Inagaki F, Amada T, Hase K, Iwanaga T, Ohno H, Fukai S (2016) Distinct Roles for the N- and C-terminal Regions of M-Sec in Plasma Membrane Deformation during Tunneling Nanotube Formation. Sci Rep 6:33548

Kimura S, Kishimoto A, Mutoh M, Takahashi-Iwanaga H, Iwanaga T (2015b) GP2-expressing cells in the conjunctiva and tear ducts of mice: identification of a novel type of cells in the squamous stratified epithelium. Biomed Res 36:263-272

Kishikawa S, Sato S, Kaneto S, Uchino S, Kohsaka S, Nakamura S, Kiyono H (2017) Allograft inflammatory factor 1 is a regulator of transcytosis in M cells. Nat Commun 8:14509

Knoop KA, Kumar N, Butler BR, Sakthivel SK, Taylor RT, Nochi T, Akiba H, Yagita H, Kiyono H, Williams IR (2009) RANKL is necessary and sufficient to initiate development of antigen-sampling $M$ cells in the intestinal epithelium. J Immunol 183:5738-5747

Kuriyama SM, Silverblatt FJ (1986) Effect of Tamm-Horsfall urinary glycoprotein on phagocytosis and killing of type I-fimbriated Escherichia coli. Infect Immun 51:193-198
Lacey DL, Timms E, Tan HL, Kelley MJ, Dunstan CR, Burgess T, Elliott R, Colombero A, Elliott G, Scully S, Hsu H, Sullivan J, Hawkins N, Davy E, Capparelli C, Eli A, Qian YX, Kaufman S, Sarosi I, Shalhoub V, Senaldi G, Guo J, Delaney J, Boyle WJ (1998) Osteoprotegerin ligand is a cytokine that regulates osteoclast differentiation and activation. Cell 93:165-176

Lee JH, Kong SK, Wu ZS, Wu Q, Choe J, Cho HY, Ha SY, Kee SH, Kim YS (2010) Class II beta-tubulin is a novel marker for human tonsillar $\mathrm{M}$ cells and follicular dendritic cells. J Oral Pathol Med 39:533-539

Lo D, Tynan W, Dickerson J, Mendy J, Chang HW, Scharf M, Byrne D, Brayden D, Higgins L, Evans C, O’Mahony DJ (2003) Peptidoglycan recognition protein expression in mouse Peyer's patch follicle associated epithelium suggests functional specialization. Cell Immunol 224:8-16

Mabbott NA, Donaldson DS, Ohno H, Williams IR, Mahajan A (2013) Microfold (M) cells: important immunosurveillance posts in the intestinal epithelium. Mucosal Imuunol 6:666-677

Maharjan S, Singh B, Jiang T, Yoon SY, Li HS, Kim G, Gu MJ, Kim SJ, Park OJ, Han SH, Kang SK, Yun CH, Choi YJ, Cho CS (2016) Systemic administration of RANKL overcomes the bottleneck of oral vaccine delivery through microfold cells in ileum. Biomaterials 84:286-300

Mantis NJ, Rol N, Corthésy B (2011) Secretory IgA's complex roles in immunity and mucosal homeostasis in the gut. Mucosal Immunol 4:603-611

Masahata K, Umemoto E, Kayama H, Kotani M, Nakamura S, Kurakawa T, Kikuta J, Gotoh K, Motooka D, Sato S, Higuchi T, Baba Y, Kurosaki T, Kinoshita M, Shimada Y, Kimura T, Okumura R, Takeda A, Tajima M, Yoshie O, Fukuzawa M, Kiyono H, Fagarasan S, Iida T, Ishii M, Takeda K (2014) Generation of colonic IgA-secreting cells in the caecal patch. Nat Commun 5:3704

Matsumura T, Sugawara Y, Yutani M, Amatsu S, Yagita H, Kohda T, Fukuoka S, Nakamura Y, Fukuda S, Hase K, Ohno H, Fujinaga Y (2015) Botulinum toxin A complex exploits intestinal M cells to enter the host and exert neurotoxicity. Nat Commun 6:6255

Mutoh M, Kimura S, Takahashi-Iwanaga H, Hisamoto M, Iwanaga T, Iida J (2016) RANKL regulates differentiation of microfold cells in mouse nasopharynx-associated lymphoid tissue (NALT). Cell Tissue Res 364:175-184

Nagashima K, Sawa S, Nitta T, Tsutsumi M, Okamura T, Penninger JM, Nakashima T, Takayanagi H (2017) Identification of subepithelial mesenchymal cells that induce $\operatorname{IgA}$ and diversify gut microbiota. Nat Immunol 18:675-682

Nakato G, Hase K, Suzuki M, Kimura M, Ato M, Hanazato M, Tobiume M, Horiuchi M, Atarashi R, Nishida N, Watarai M, Imaoka K, Ohno H (2012) Brucella abortus exploits a cellular prion protein on intestinal $\mathrm{M}$ cells as an invasive receptor. J Immunol 189:1540-1544

Nochi T, Yuki Y, Matsumura A, Mejima M, Terahara K, Kim DY, Fukuyama S, Iwatsuki-Horimoto K, Kawaoka Y, Kohda T, Kozaki S, Igarashi O, Kiyono H (2007) A novel M cell-specific carbohydrate targeted mucosal vaccine effectively induces antigen-specific immune responses. J Exp Med 204:2789-2796

Oeckinghaus A, Hayden MS, Ghosh S (2011) Crosstalk in NF-кB signaling pathways. Nat Immunol 12:695-708

Ohishi I, Sugii S, Sakaguchi G (1977) Oral toxicities of Clostridium botulinum toxins in response to molecular size. Infect Immun 16:107-109

Ohsawa K, Imai Y, Kanazawa H, Sasaki Y, Kohsaka S (2000) Involvement of Iba1 in membrane ruffling and phagocytosis of macrophages/microglia. J Cell Sci 113:3073-3084

Okamoto K, Nakashima T, Shinohara M, Negishi-Koga T, Komatsu N, Terashima A, Sawa S, Nitta T, Takayanagi H (2017) 
Osteoimmunology: the conceptual framework unifying the immune and skeletal systems. Physiol Rev 97:1295-1349

Rindler MJ, Hoops TC (1990) The pancreatic membrane protein GP-2 localizes specifically to secretory granules and is shed into the pancreatic juice as a protein aggregate. Eur J Cell Biol 53:154-163

Rios D, Wood MB, Li J, Chassaing B, Gewirtz AT, Williams IR (2016) Antigen sampling by intestinal $M$ cells is the principal pathway initiating mucosal IgA production to commensal enteric bacteria. Mucosal Immunol 9:907-916

Rouch JD, Scott A, Lei NY, Solorzano-Vargas RS, Wang J, Hanson EM, Kobayashi M, Lewis M, Stelzner MG, Dunn JC, Eckmann L, Martín MG (2016) Development of functional microfold (M) cells from intestinal stem cells in primary human enteroids. PLoS ONE 11:e0148216

Sakaguchi G (1982) Clostridium botulinum toxins. Pharmacol Ther 19:165-194

Sato T, Vries RG, Snippert HJ, van de Wetering M, Barker N, Stange DE, van Es JH, Abo A, Kujala P, Peters PJ, Clevers H (2009) Single Lgr5 stem cells build crypt-villus structures in vitro without a mesenchymal niche. Nature 459:262-265

Sato S, Kaneto S, Shibata N, Takahashi Y, Okura H, Yuki Y, Kunisawa J, Kiyono H (2013) Transcription factor Spi-B-dependent and -independent pathways for the development of Peyer's patch M cells. Mucosal Immunol 6:838-846

Scheffer RC, Poort C, Slot JW (1980) Fate of the major zymogen granule membrane-associated glycoproteins from rat pancreas. A biochemical and immunocytochemical study. Eur J Cell Biol 23:122-128

Shima H, Watanabe T, Fukuda S, Fukuoka S, Ohara O, Ohno H (2014) A novel mucosal vaccine targeting Peyer's patch M cells induces protective antigen-specific IgA responses. Int Immunol 26:619-625

Shinkura R, Kitada K, Matsuda F, Tashiro K, Ikuta K, Suzuki M, Kogishi K, Serikawa T, Honjo T (1999) Alymphoplasia is caused by a point mutation in the mouse gene encoding Nf-kappa b-inducing kinase. Nat Genet 22:74-77

Takano K, Kojima T, Ogasawara N, Go M, Kikuchi S, Ninomiya T, Kurose M, Koizumi J, Kamekura R, Murata M, Tanaka S, Chiba H, Himi T, Sawada N (2008) Expression of tight junction proteins in epithelium including Ck20-positive M-like cells of human adenoids in vivo and in vitro. $\mathrm{J}$ Mol Histol 39:265-273

Taylor RT, Patel SR, Lin E, Butler BR, Lake JG, Newberry RD, Williams IR (2007) Lymphotoxin-independent expression of TNFrelated activation-induced cytokine by stromal cells in cryptopatches, isolated lymphoid follicles, and Peyer's patches. J Immunol 178:5659-5667

Terahara K, Yoshida M, Igarashi O, Nochi T, Pontes GS, Hase K, Ohno H, Kurokawa S, Mejima M, Takayama N, Yuki Y, Lowe AW,
Kiyono H (2008) Comprehensive gene expression profiling of Peyer's patch M cells, villous M-like cells, and intestinal epithelial cells. J Immunol 180:7840-7846

Totsuka T, Kanai T, Nemoto Y, Tomita T, Okamoto R, Tsuchiya K, Nakamura T, Sakamoto N, Akiba H, Okumura K, Yagita H, Watanabe M (2009) RANK-RANKL signaling pathway is critically involved in the function of CD4+ CD25+ regulatory T cells in chronic colitis. J Immunol 182:6079-6087

Utans U, Arceci RJ, Yamashita Y, Russell ME (1995) Cloning and characterization of allograft inflammatory factor-1: a novel macrophage factor identified in rat cardiac allografts with chronic rejection. J Clin Invest 95:2954-2962

Verbrugghe P, Kujala P, Waelput W, Peters PJ, Cuvelier CA (2008) Clusterin in human gut-associated lymphoid tissue, tonsils, and adenoids: localization to $\mathrm{M}$ cells and follicular dendritic cells. Histochem Cell Biol 129:311-320

Walsh MC, Choi Y (2014) Biology of the RANKL-RANK-OPG system in immunity, bone, and beyond. Front Immunol 5:511

Wiedemann A, Linder S, Grassl G, Albert M, Autenrieth I, Aepfelbacher M (2001) Yersinia enterocolitica invasin triggers phagocytosis via beta1 integrins, CDC42Hs and WASp in macrophages. Cell Microbiol 3:693-702

Wolf FW, Sarma V, Seldin M, Drake S, Suchard SJ, Shao H, O'Shea KS, Dixit VM (1994) B94, a primary response gene inducible by tumor necrosis factor-alpha, is expressed in developing hematopoietic tissues and the sperm acrosome. J Biol Chem 269:3633-3640

Wong BR, Josien R, Lee S, Sauter B, Li H, Steinman RM, Choi Y (1997) TRANCE (tumor necrosis factor [TNF]-related activationinduced cytokine), a new TNF family member predominantly expressed in T cells, is a dendritic cell-specific survival factor. J Exp Med 186:2075-2080

Wood MB, Rios D, Williams IR (2016) TNF- $\alpha$ augments RANKLdependent intestinal $\mathrm{M}$ cell differentiation in enteroid cultures. Am J Physiol Cell Physiol 311:C498-C507

Yasuda H, Shima N, Nakagawa N, Yamaguchi K, Kinosaki M, Mochizuki S, Tomoyasu A, Yano K, Goto M, Murakami A, Tsuda E, Morinaga T, Higashio K, Udagawa N, Takahashi N, Suda T (1998) Osteoclast differentiation factor is a ligand for osteoprotegerin/ osteoclastogenesis-inhibitory factor and is identical to TRANCE/ RANKL. Proc Natl Acad Sci USA 95:3597-3602

Yoo MK, Kang SK, Choi JH, Park IK, Na HS, Lee HC, Kim EB, Lee NK, Nah JW, Choi YJ, Cho CS (2010) Targeted delivery of chitosan nanoparticles to Peyer's patch using M cell-homing peptide selected by phage display technique. Biomaterials 31:7738-7747 